\title{
High-Resolution Terahertz Spectrometer
}

\author{
Gregor Klatt, Raphael Gebs, Hanjo Schäfer, Michael Nagel, Christof Janke, Albrecht Bartels, and Thomas Dekorsy
}

\begin{abstract}
Terahertz time-domain spectroscopy (THz-TDS) based on high-speed asynchronous optical sampling (ASOPS) with two offset-locked $\mathrm{GHz}$ femtosecond lasers requires no mechanical time-delay scanner. Consequently, measurements with 1-GHz frequency resolution are performed at intrinsically high scan rates in the multikilohertz range. This is at least one order, in most cases several orders of magnitude faster than conventional approaches employing mechanical time-delay scanners. We report a system offering a unique combination of high-frequency resolution $(1 \mathrm{GHz})$ and high scan rate $(2 \mathrm{kHz})$ with a spectral coverage of more than $6 \mathrm{THz}$. Its capabilities for high-precision spectroscopy are demonstrated by measuring the absorption spectrum of a mixture of $\mathrm{H}_{2} \mathrm{O}, \mathrm{D}_{2} \mathrm{O}$, and hydrogen deuterium oxide (HDO) vapor. $\mathrm{H}_{2} \mathrm{O}$ and $\mathrm{HDO}$ vapor absorption spectra are accurately tabulated in databases. However, $\mathrm{D}_{2} \mathrm{O}$ absorption data are rare, because of residual $\mathrm{H}_{2} \mathrm{O}$ and $\mathrm{HDO}$ often present when measuring pure $\mathrm{D}_{2} \mathrm{O}$. Here, we present a high-resolution absorption spectrum of $\mathrm{D}_{2} \mathrm{O}$ vapor numerically extracted from the absorption spectrum of the three-component mixture. In addition, we show that the high spectral resolution of the ASOPS THz-TDS system provides benefits in the analysis of frequency-selective surface sensors, which are promising candidates for biosensing applications in the THz regime.
\end{abstract}

Index Terms-Submillimeter wave spectroscopy, submillimeter wave technology, ultrafast optics, water.

\section{INTRODUCTION}

$\mathbf{T}$ ERAHERTZ time-domain spectroscopy (THz-TDS) has emerged as an important tool for a variety of applications in $\mathrm{THz}$ science and technology. Prominent examples are drug and food inspection, detection of explosives, label-free DNA analysis, or environmental gas analysis and sensing [1]-[3] THz-TDS is based on ultrashort laser pulses from a modelocked laser for the generation of an ultrashort $\mathrm{THz}$ pulse and a delayed probe pulse for phase-sensitive detection of the radiation. THz-TDS systems meanwhile cover a large frequency

This work was supported in part by the Landesstiftung BadenWüttemberg in part by the Ministry of Science Research and the Arts of Baden-Württemberg, and in part by the Deutsche Forschungsgemeinschaft.

G. Klatt, R. Gebs, H. Schäfer, and T. Dekorsy are with the Department of Physics and the Center for Applied Photonics, University of Konstanz, D-78457 Konstanz, Germany (e-mail: gregor.klatt@uni-konstanz.de; raphael gebs@uni-konstanz.de; hanjo.schaefer@uni-konstanz.de; thomas.dekorsy@ uni-konstanz.de).

M. Nagel is with the Institute of Semiconductor Electronics, Rheinisch Westfälische Technische Hochschule Aachen University, D-52074 Aachen, Germany (e-mail: nagel@iht.rwth-aachen.de).

C. Janke and A. Bartels are with Gigaoptics GmbH, 78462 Konstanz Germany, and also with the Department of Physics and the Center for Applied Photonics, University of Konstanz, D-78457 Konstanz, Germany (e-mail: christof.janke@uni-konstanz.de; albrecht.bartels@uni-konstanz.de).

Digital Object Identifier 10.1109/JSTQE.2010.2047635 range from a few tens of $\mathrm{GHz}$ into the near-infrared [4]-[7]. One drawback of conventional THz-TDS systems is the use of mechanical delay stages to realize the time delay between the THz pulse and the optical detection pulse. This leads to an inherently low acquisition rate for $\mathrm{THz}$ transients. For the analysis of molecular absorption spectra in the gas phase, a high spectral resolution in the $1-\mathrm{GHz}$ range, and thus a time delay of $1 \mathrm{~ns}$ is required. To this end, a conventional THz-TDS system needs a mechanical delay stage with a travel distance of $15 \mathrm{~cm}$. The time required for accelerating and decelerating the stage between approximately 10000 data points and to average out laser noise typically leads to a total acquisition time in the range of a few tens of minutes. Hence, applications, where the acquisition of a THz trace should be completed within a few seconds or even milliseconds, are impossible. Examples for such applications are the fast readout of resonant sensors [8], the observation of conformational dynamics of biomolecules [9], or experiments under rapidly changing external variables, such as spectroscopy in a millisecond pulsed magnetic field [10]. Recently, different systems have been proposed, which operate at higher scan rates, e.g., rotating mirrors with up to $400-\mathrm{Hz}$ scan rate and up to $1 \mathrm{~ns}$ time delay [11], [12]. While these systems are advantageous compared to linear stages, drawbacks like noise resulting from masses rotating at $>10000 \mathrm{r} / \mathrm{min}$ on the optical table and a scan rate significantly below $1 \mathrm{kHz}$, where common lasers exhibit the bulk of their amplitude noise, limit their utility. In addition, it should be mentioned that mechanical delay stages are a source of frequency calibration errors due to manufacturing tolerances and limited alignment accuracy.

\section{ASYNCHRONOUS OPTICAL SAMPLING}

Asynchronous optical sampling (ASOPS) has been introduced with $81-\mathrm{MHz}$ repetition rate picosecond dye lasers in the late 1980s and is a TDS technique that does not require a mechanical delay stage, thus avoiding the potential drawbacks discussed earlier [13]. Our high-speed implementation of ASOPS operates with two femtosecond lasers with repetition rates close to $1 \mathrm{GHz}$. The repetition rates are stabilized with a constant offset frequency $\Delta f_{R}$ in the range of a few kilohertz [14], [15]. As a result of this offset frequency, the time delay between pairs of pulses from the lasers is repetitively ramped between zero and the inverse laser repetition rate (1 ns) at a scan rate equal to $\Delta f_{R}$. Fig. 1 depicts the ASOPS principle for the case that one laser serves as pump for the $\mathrm{THz}$ generation, and the other laser is used for electrooptic sampling of the $\mathrm{THz}$ transient under the assumption that $\Delta f_{R}=10 \mathrm{kHz}$. The key advantage of high-speed ASOPS is the scan rate of several kilohertz, which is impossible with mechanical systems. The scan rate allows completing the acquisition of a time-domain trace before technical noise of common femtosecond lasers with significant Fourier 


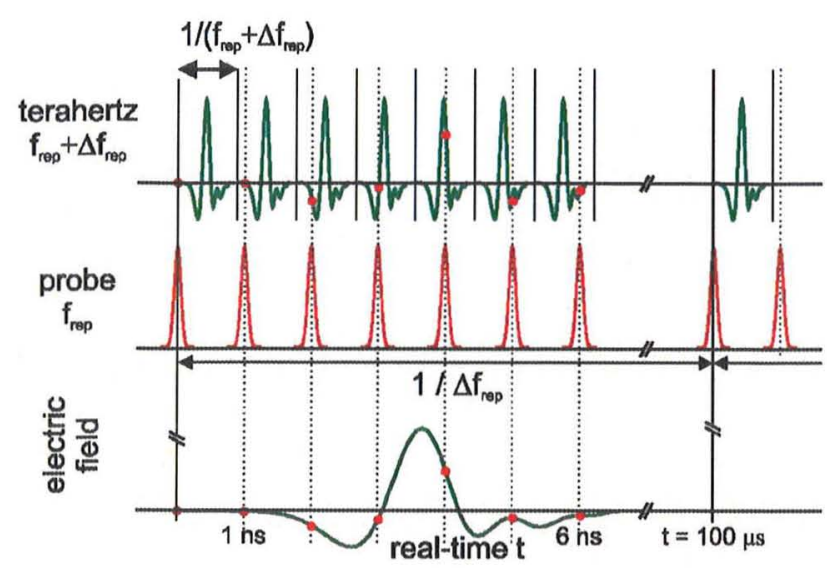

Fig. 1. Sketch of the ASOPS principle. The pump laser generates the $\mathrm{THz}$ pulses (upper row) at a repetition rate of $f_{\text {rep }}+\Delta f_{\text {rep }}$, which are sampled by the probe laser (middle row) with $f_{\text {rep }}$. The bottom row indicates the $\mathrm{THz}$ waveform after the mapping through the sample pulse.

content in the acoustic regime up to $1 \mathrm{kHz}$ can affect the signal. Thus, with high-speed ASOPS, measurements at the shot-noise limit are possible without use of lock-in amplifiers or other noise suppression techniques [14].

As depicted in Fig. 2, for the realization of a THz-TDS system, one laser is used to generate the $\mathrm{THz}$ radiation, the other laser is used to detect the $\mathrm{THz}$ transient in the same geometry as in a conventional THz-TDS setup, e.g., via electrooptic detection [4]-[7]. The THz electric field is mapped onto the polarization of the detection laser beam, which is measured as intensity change in a polarization sensitive detection scheme. These intensity changes are digitized as a function of real time $t$ and recalibrated to a time-delay scale $\tau$, using a factor $\Delta f_{R} / f_{R}$, where $f_{R}$ is the repetition rate of the $\mathrm{THz}$ generation laser.

In the last three years, the performance of our high-speed ASOPS THz-TDS spectroscopy approach has improved significantly [14]-[16]. While the first implementation had a spectral coverage of $0.5 \mathrm{THz}$, the latest implementation of the high-speed ASOPS THz spectrometer achieves a spectral coverage of more than $6.5 \mathrm{THz}$, corresponding to a time resolution of better than $50 \mathrm{fs}$ and a spectral resolution of $1 \mathrm{GHz}$.

\section{THz SPECTROMETER}

The THz-TDS system is sketched in Fig. 2. Core elements are two $1-\mathrm{GHz}$ repetition rate Ti:sapphire lasers sharing a common housing milled from a solid aluminum block that are operated in a master-slave configuration. The repetition rate of the master laser is free running and the slave laser repetition rate is stabilized with an offset $\Delta f$, using a phase-locked loop. A repetition rate difference $\Delta f$ of $2 \mathrm{kHz}$ in combination with the $1-\mathrm{GHz}$ repetition rate and the $100 \mathrm{MHz}$ bandwidth of the photodetector and the analog-to-digital (A/D) converter yields a data point spacing of $20 \mathrm{fs}$, which is sufficient to support the 45-fs time resolution as given by the duration of the laser pulses [17].

Both lasers deliver about $800 \mathrm{~mW}$ of average output power at center wavelengths of $825 \mathrm{~nm}$ and have a pulse duration of $\approx 45 \mathrm{fs}$. A small portion of the output power of each laser is split off with beamsplitters and is focused onto $10-\mathrm{GHz}$ bandwidth photodiodes in order to detect the repetition rates and their harmonics. The photodiode signals are used to phase-coherently generate an error signal, representing deviations of the repetition rate-offset $\Delta f$ from the desired value $\Delta f_{\text {set }}$. The error signal is amplified and low-pass filtered with a loop filter and further amplified by use of a high-voltage amplifier to control the slave repetition rate. This is accomplished via a weight-reduced cavity mirror mounted onto a high-bandwidth piezoelectric transducer for adjusting the cavity length of the slave laser. Details of the offset stabilization have been outlined previously [17]. The A/D converter is triggered by a cross-correlation signal between master and slave laser generated via two-photon absorption in a GaP photodiode, using split-off beams from both lasers with $100-\mathrm{mW}$ average power.

Fig. 3(a) shows the first $40 \mathrm{ps}$ of a 1-ns-long THz transient. The presented data were acquired in $9 \mathrm{~min}$ and the spectrometer was purged with dry air. The main $\mathrm{THz}$ pulse is generated by an optical pump pulse of $45 \mathrm{fs}$ duration and $55-\mu \mathrm{m}$ spot size (fullwidth at half maximum) with an average power of $550 \mathrm{~mW}$ on a large area photoconductive $\mathrm{THz}$ emitter. The emitter is dc-biased with $10 \mathrm{~V}$, which corresponds to an electric field of $20 \mathrm{kV} / \mathrm{cm}$ for the acceleration of carriers in the GaAs substrate. Details of the emitter are described elsewhere [18]. Internal reflections of the main $\mathrm{THz}$ pulse in the 400- $\mu$ m-thick GaP detector crystal and in the $645-\mu$ m-thick GaAs emitter substrate lead to satellite signals at 9 and 16 ps and combinations of these values at later time delays, respectively. The satellites do not influence absorption measurements, because they are equally present in the reference measurement and in the sample measurement and thus divide out in the Fourier domain. Fig. 3(b) shows the corresponding numerical Fourier transform of the full time-domain data. The high-frequency modulation on the spectrum stems from the multiple reflections of the main $\mathrm{THz}$ pulse in the time domain. The spectral coverage above the noise floor ranges from 0.2 to $6.5 \mathrm{THz}$. Fast oscillations present on the signal after the main THz pulse [see Fig. 3(a) inset] arise from the reduced propagation velocity of phonon-polaritons in the GaAs emitter substrate at frequencies close to the transverseoptical (TO) phonon [15], [19], [20].

The limitation of the spectrometer bandwidth to less than $7 \mathrm{THz}$ originates from the nonlinear response function of the $\mathrm{GaP}$ detection crystal used for electrooptic detection via the Pockels effect. In the $\mathrm{THz}$ frequency range, an electronic part as well as an ionic part contributes to the second-order susceptibility $\chi^{2}$. The ratio between both parts is described by the Faust-Henry coefficient [21]. For many semiconductors, such as GaP, the Faust-Henry coefficient is negative. This expresses that both contributions subtract from each other for frequencies below the TO phonon frequency. In the case of GaP, the electronic and ionic part cancel out in the frequency range around $7 \mathrm{THz}$ [19], [21], [22]. While the response of GaP recovers beyond the minimum around $7 \mathrm{THz}$, the spectral coverage of the spectrometer is still limited to frequencies below $8 \mathrm{THz}$. This is due to the TO phonon of the GaAs emitter substrate, which could be circumvented by either using the emitter in reflection geometry or by thinning down the emitter substrate [23]. 


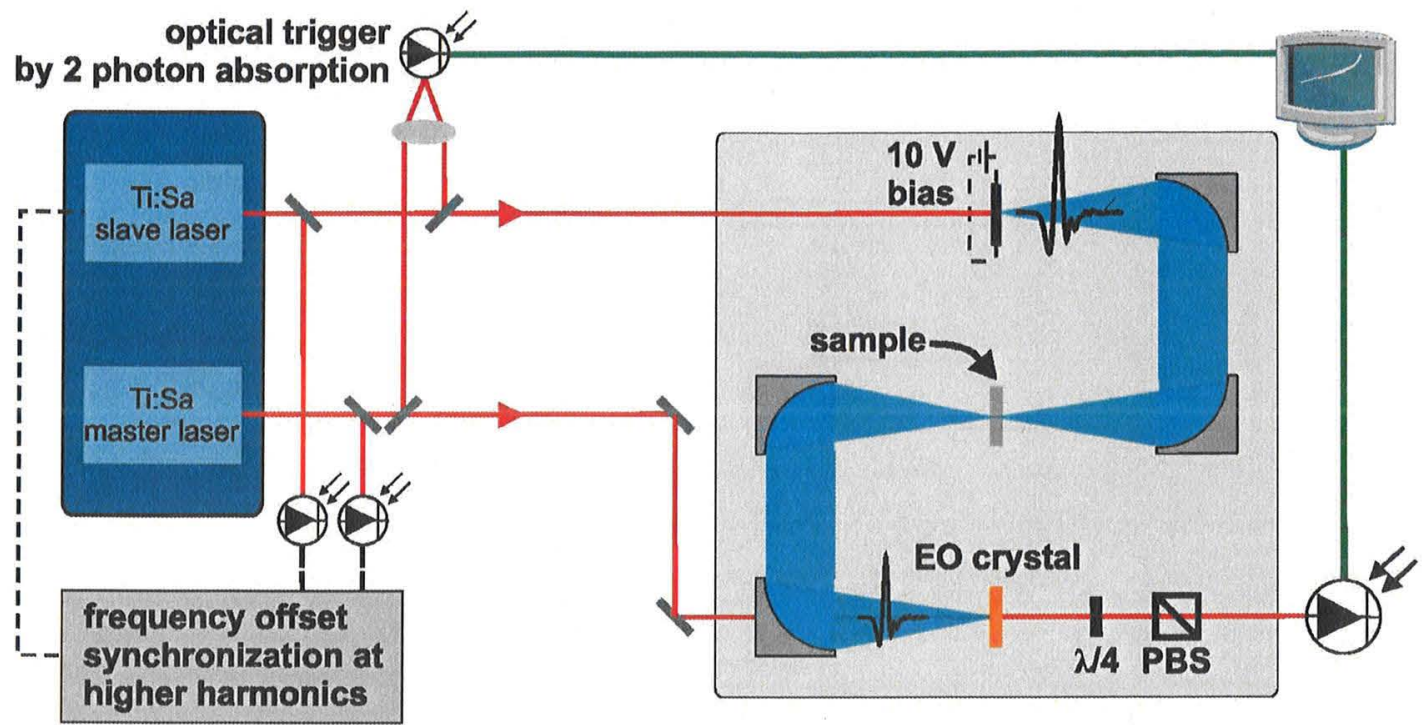

Fig. 2. Setup of the high-speed ASOPS THz spectrometer.
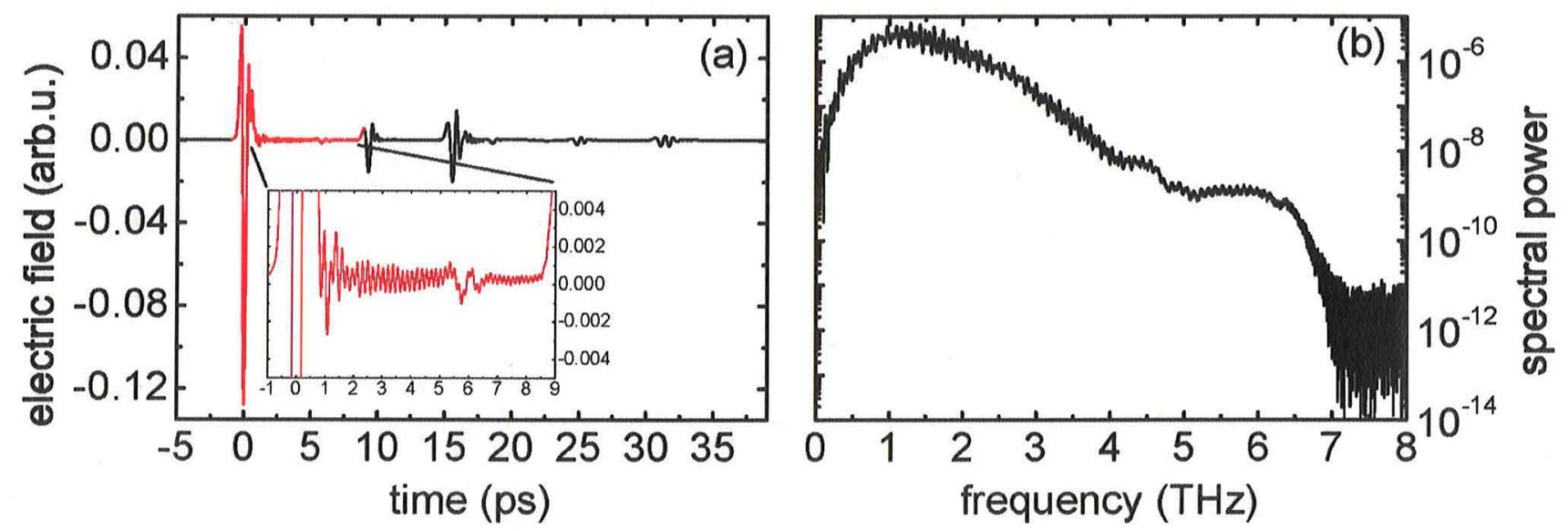

Fig. 3. (a) THz transient with multiple reflections of the THz pulse in the THz emitter substrate and in the GaP detection crystal. The inset shows a zoomin into the first picoseconds after the main $\mathrm{THz}$ pulse. The high-frequency components stem from the phonon-polariton propagation in the emitter substrate. (b) Corresponding Fourier transform of the $\mathrm{THz}$ transient shown in (a) on a logarithmic scale.

Beyond $8 \mathrm{THz}$, the 45 -fs pulse duration of the $1 \mathrm{GHz}$ Ti:sapphire laser are the limiting factor.

\section{SYSTEM ACCURACY}

The frequency accuracy of a $\mathrm{THz}$ spectrometer based on a THz-TDS system is determined by the accuracy of the timedelay scale $\tau$. For a conventional THz-TDS system, the frequency accuracy is entirely given by the calibration and alignment of the delay stage. For a system based on high-speed ASOPS, the time delay is given as $\tau=t \times \Delta f_{R} / f_{R}$, and its accuracy is thus determined by that of the spectrum analyzer or frequency counter used to measure the repetition rates and the system clock of the A/D converter that defines the real-time scale $t$. The accuracy of these instruments can be as good as that of a cesium atomic clock $\left(10^{-15}\right)$ or a more readily available commercial GPS-referenced Rubidium reference $\left(10^{-12}\right)$. However, in the ASOPS THz-TDS spectrometer described here a lower cost oven stabilized crystal oscillator is used with a relative frequency uncertainty of approximately $10^{-7}$. Another potential factor, which might influence the accuracy of a highspeed ASOPS system, are deviations of the true $\Delta f_{R} / f_{R}$ value from the nominal value that occur during a measurement cycle [17]. These deviations may be in phase with the trigger signal, i.e., they repeat for each measurement, such that they do not average out. Modulations of $\Delta f_{R}$ due to imperfections in the offset-locking electronics can cause such systematic deviations. In a cross-correlation measurement, the mean relative deviation of $\Delta f_{R} / f_{R}$ from the nominal value was determined to $9.8 \times 10^{-5}$, the associated maximum relative uncertainty of the frequency calibration is $1.7 \times 10^{-4}$ [17].

\section{ATMOSPHERIC WATER VAPOR $\left(\mathrm{H}_{2} \mathrm{O}\right)$}

One of the most suited samples to test the spectral accuracy of a THz-TDS system is atmospheric water vapor [24]. Between 


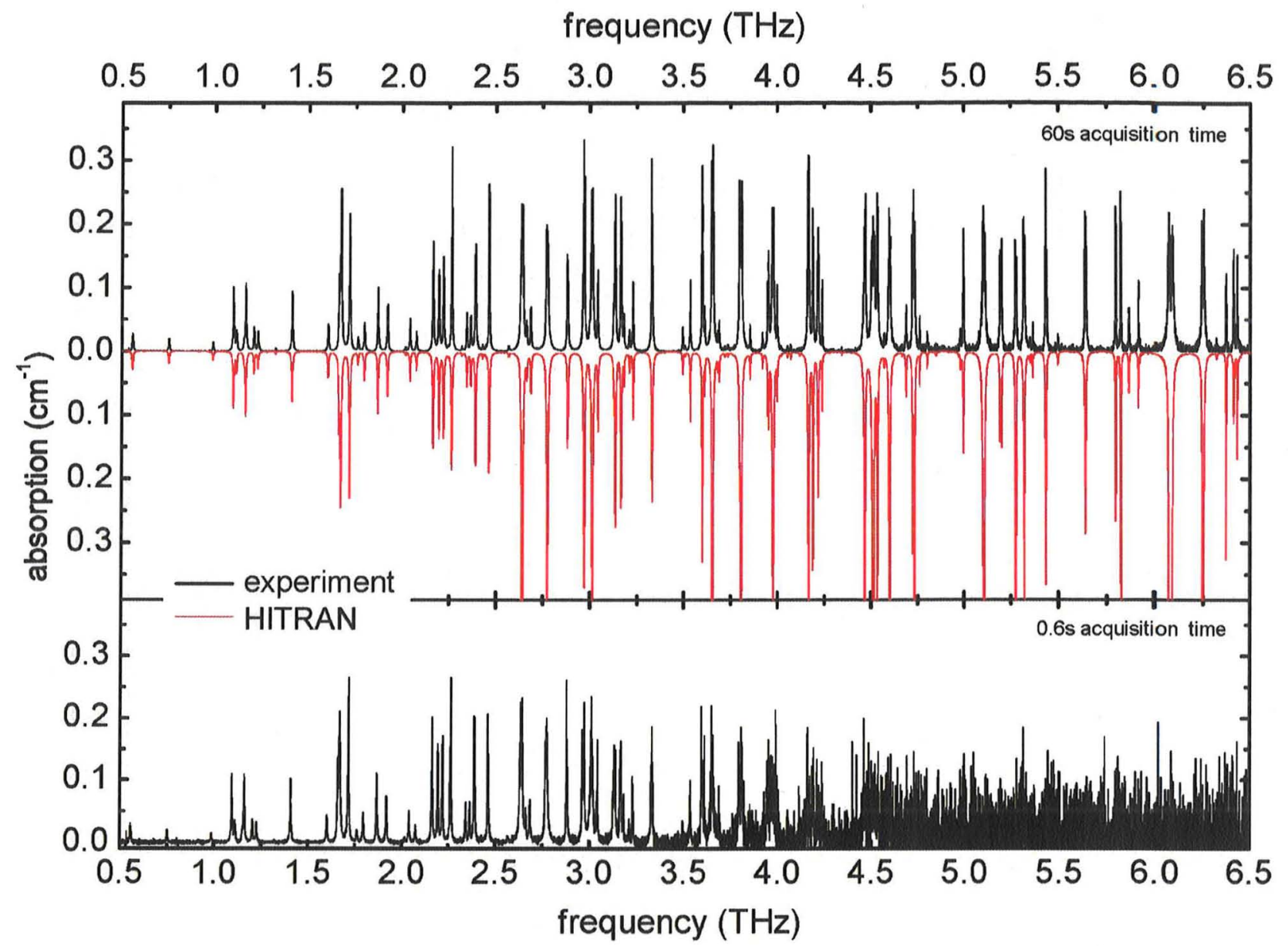

Fig. 4. Measured (black) and calculated (red) absorption spectra of atmospheric water vapor acquired in (top) $60 \mathrm{~s}$ and (bottom) $0.6 \mathrm{~s}$. The calculated spectrum is based on the HITRAN database.

0.2-6.5 THz more than 1000 absorption lines exist, which are well known from experimental investigations and theoretical calculations. These lines are documented in the HITRAN and JPL databases [25]-[27]. For the HITRAN database of water vapor data, the reported frequency error is between $300 \mathrm{kHz}$ and $3 \mathrm{MHz}$. Hence, it is possible to test the $\mathrm{THz}$ spectrometer at this accuracy level. At atmospheric conditions, the width of each absorption line lies in the range of $10 \mathrm{GHz}$, which is well matched to the $1 \mathrm{GHz}$ resolution of the $\mathrm{THz}$ spectrometer determined by the $1 \mathrm{~ns}$ observation window. At the top of Fig. 4 , the comparison between a measured and calculated water vapor absorption spectrum is plotted. The acquisition time for the transient with water vapor was $60 \mathrm{~s}$, the reference data are the data averaged over $9 \mathrm{~min}$, as shown in Fig. 3. At this point, we emphasize that a long reference measurement does not affect the high-speed capability of the system, but rather helps improving the quality of subsequently acquired absorption data calculated from traces with much shorter acquisition times (e.g., $0.6 \mathrm{~s}$, see later on and Fig. 4). The calculated spectrum is based on data from the latest version of the HITRAN database [27]. The formulas for the calculation of the full-spectral lineshapes can be found in reference [26]. During the $60 \mathrm{~s}$ measurement, the ambient parameters were kept constant (temperature $20^{\circ} \mathrm{C}$, pressure $1015 \mathrm{hPa}$, relative humidity $26 \%$ ).

From 0.2 to $2.5 \mathrm{THz}$, the measured and calculated absorption spectra agree very well quantitatively in frequency and amplitude. Above $2.5 \mathrm{THz}$, the reduced dynamics range of the spectrometer lead to deviations in the measured absorption strength. Absorption lines, which exceed $0.15 \mathrm{~cm}^{-1}$ cannot be fully resolved (see Fig. 5). However, the frequency of each absorption line can still be evaluated with high precision. To analyze the water vapor absorption data in more detail, all measured absorption lines from 0.2 to $6.5 \mathrm{THz}$ were fitted with a Lorentzian lineshape given by

$$
\alpha(\nu)=S \rho \frac{1}{\pi} \frac{\gamma}{\gamma^{2}+\left(\nu-\nu_{0}\right)^{2}}
$$

where $\alpha$ is the absorption cocfficient in $\mathrm{cm}^{-1}, \nu$ is the wavenumber in $\mathrm{cm}^{-1}, S$ is the line intensity in $\mathrm{cm}^{-1} /\left(\right.$ molecule $\left.\cdot \mathrm{cm}^{-2}\right)$, $\rho$ is the number of $\mathrm{H}_{2} \mathrm{O}$ molecules per $\mathrm{cm}^{3}, \gamma$ is the pressuredependent half-width half maximum (HWHM) in $\mathrm{cm}^{-1}$, and $\nu_{0}$ is the pressure corrected line position in $\mathrm{cm}^{-1}$. For more details, see [26]. An automatic numerical routine analyzes the experimental data: The routine extracts all line positions and performs 


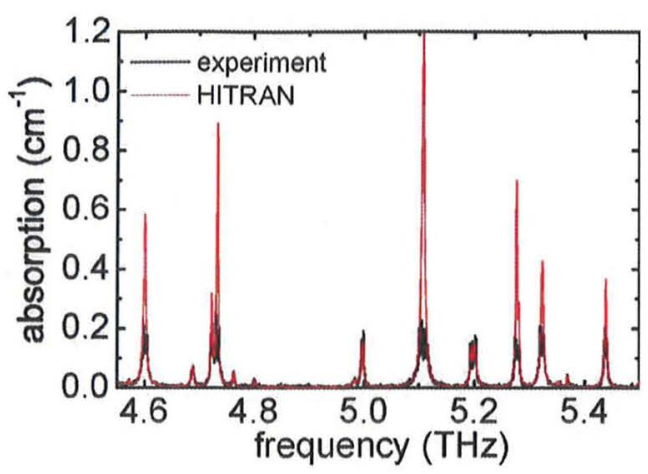

Fig. 5. Detailed view of the absorption spectra around $5 \mathrm{THz}$. The noisy part of the experimental data is deleted when performing the fits.

a Lorentzian lineshape fit for every absorption line. For lines with a frequency greater than $2.5 \mathrm{THz}$ and absorption strength greater than $0.15 \mathrm{~cm}^{-1}$, the shape is not fully resolved. Hence, the noisy center part of these absorption lines is removed, and the fit is based on the wings of the lines.

In the range from 0.2 to $6.5 \mathrm{THz}, 147$ lines can be found in the HITRAN database with intensities larger than $3 \times 10^{-21} \mathrm{~cm}^{-1} /\left(\right.$ molecule $\left.\cdot \mathrm{cm}^{-2}\right)$. This intensity gives the lower detection limit of the $\mathrm{THz}$ spectrometer in the range from 0.2 to $6.5 \mathrm{THz}$. Eighty-seven lines are evaluated by the mentioned numerical routine. The remaining 60 lines are either too close to a neighboring line and hence lead to a nonconverging fit or their intensity is too weak to be detected numerically. The mean deviation of the line positions $\nu_{0}$ from the HITRAN data over all fitted lines is $142 \mathrm{MHz}$. The mean relative deviation is $5.7 \times 10^{-5}$. The largest deviation of any line is $461 \mathrm{MHz}$, corresponding to a relative deviation of $2.5 \times 10^{-4}$. These errors are consistent with the earlier estimated effect of a systematic calibration uncertainty of the time-delay versus real-time scale due to imperfections in the locking electronics. The errors of our measurements of temperature $( \pm 1 \mathrm{~K})$, pressure $( \pm 5 \mathrm{hPa})$, and humidity ( $\pm 2 \%$ relative humidity) cause only a negligible uncertainty (maximum $\pm 1.5 \mathrm{MHz}$ ) in the calculation from the HITRAN database and are not significant in the evaluation of the frequency error.

The averaged relative deviation of the line intensity $S$ from the calculated spectrum based on the HITRAN data is $6.0 \%$ for the fully resolved lines below $2.5 \mathrm{THz}$ and increases to $12.3 \%$ when all evaluated lines are considered. This is consistent with the $6 \%$ uncertainty in the calculated line intensity due to the mentioned errors of temperature, pressure, and humidity and additional errors expected from the limited dynamic range of the spectrometer above $2.5 \mathrm{THz}$.

We have repeated the same evaluation for data acquired with $0.6 \mathrm{~s}$ acquisition time, as shown in the bottom panel of Fig. 4. The mean and maximum deviations from the HITRAN database increase only slightly to 164 and $468 \mathrm{MHz}$, respectively. While these values are similar to the data at 60-s acquisition time, the available spectral coverage was obviously reduced to $3.5 \mathrm{THz}$ due to a reduced SNR above this value.

\section{HEAVY WATER VAPOR $\left(\mathrm{D}_{2} \mathrm{O}\right)$}

The natural concentration of ${ }^{1} \mathrm{H}_{2}^{6} \mathrm{O}$ water molecules compared to all other isotopes of water is $99.73 \%$ [28]. $\mathrm{D}_{2} \mathrm{O}$ absorption data are rare due to the difficulty in establishing experimental conditions for measuring pure $\mathrm{D}_{2} \mathrm{O}$ vapor, since it is hardly possible to remove $\mathrm{H}_{2} \mathrm{O}$ molecules completely from the sample volume and maintain pure $\mathrm{D}_{2} \mathrm{O}$ vapor in the cell [29], [30].

The replacement of $\mathrm{H}_{2} \mathrm{O}$ vapor with $\mathrm{D}_{2} \mathrm{O}$ vapor is very difficult. One possibility is to evacuate the sample volume and subsequently insert liquid $\mathrm{D}_{2} \mathrm{O}$, which immediately evaporates. A problem of this method is the immediate contamination of $\mathrm{D}_{2} \mathrm{O}$ molecules with $\mathrm{H}_{2} \mathrm{O}$ molecules that are left adherent to the chamber walls. These $\mathrm{H}_{2} \mathrm{O}$ molecules interchange with the inserted $\mathrm{D}_{2} \mathrm{O}$ due to similar partial vapor pressures. Also, the two water species interchange $\mathrm{H}$ and $\mathrm{D}$ atoms very rapidly, and the third water species hydrogen deuterium oxide (HDO) is formed [31].

The HITRAN database only contains data for $\mathrm{H}_{2} \mathrm{O}$ - and $\mathrm{HDO}-$ vapor, but there are no data available for $\mathrm{D}_{2} \mathrm{O}$ [27]. An additional source for water vapor data is the JPL database [25]. From the HITRAN database, it is possible to reconstruct the full experimental absorption spectrum for $\mathrm{H}_{2} \mathrm{O}$ and $\mathrm{HDO}$ (see Figs. 4 and 5), but the JPL database only contains the line positions and the integrated intensities. From these data, it is not possible to reconstruct a full absorption spectrum due to missing lineshape data, i.e., the HWHM at a given pressure and temperature of each line.

Hence, Fig. 6 compares only the line positions of $\mathrm{H}_{2} \mathrm{O}$, $\mathrm{HDO}$, and $\mathrm{D}_{2} \mathrm{O}$ from the JPL database with the experimental absorption spectrum. From the experimental water vapor mixture data, it is possible to calculate a $\mathrm{D}_{2} \mathrm{O}$ absorption spectrum by subtracting calculated absorption spectra of $\mathrm{H}_{2} \mathrm{O}$ and $\mathrm{HDO}$ based on the full information of the HITRAN database. The best suppression of $\mathrm{H}_{2} \mathrm{O}$ and $\mathrm{HDO}$ lines is achieved for an assumed $\mathrm{H}_{2} \mathrm{O}$ humidity of $1.4 \%$ and a HDO humidity of $4.5 \%$. The optimal humidity values are found by iteratively varying these values and subsequent subtraction of the calculated spectrum from the experimental one, until the best result of $\mathrm{H}_{2} \mathrm{O}$ and HDO line suppression is achieved (see Fig. 7). The high accuracy of the ASOPS system with respect to frequency and amplitude enables this simple procedure without further data processing. From Fig. 7, it is obvious that negative absorption peaks result for some removed $\mathrm{H}_{2} \mathrm{O}$ and $\mathrm{HDO}$ lines. These residual errors are most pronounced in the vicinity of strong $\mathrm{H}_{2} \mathrm{O}$ lines and strong HDO lines and are caused by our remaining uncertainty in the partial humidity values. It should be pointed out that the HITRAN database contains the absorption data of atmospheric water vapor with a natural HDO concentration of $0.031 \%$. Thus, the HDO part of the calculated absorption spectrum must be multiplied by $\sim 3200$ to obtain results for pure HDO vapor.

From the $\mathrm{H}_{2} \mathrm{O}$ and $\mathrm{HDO}$ concentration, it is possible to calculate the $\mathrm{D}_{2} \mathrm{O}$ concentration. By setting up the rate equation for the generation and elimination of one water species, the concentration of $\mathrm{D}_{2} \mathrm{O}$ is given by $n_{\mathrm{D} 2 \mathrm{O}}=n_{\mathrm{HDO}}^{2} / 4 n_{\mathrm{H} 2 \mathrm{O}}$. For the humidity values $\mathrm{H}_{2} \mathrm{O}$ and $\mathrm{HDO}(1.4 \%$ and $4.5 \%$ humidity, respectively), this yields a $\mathrm{D}_{2} \mathrm{O}$ humidity of $3.6 \%$. Hence, the ratio of $\mathrm{H}_{2} \mathrm{O}: \mathrm{HDO}: \mathrm{D}_{2} \mathrm{O}$ is about 1:3.2:2.5. 


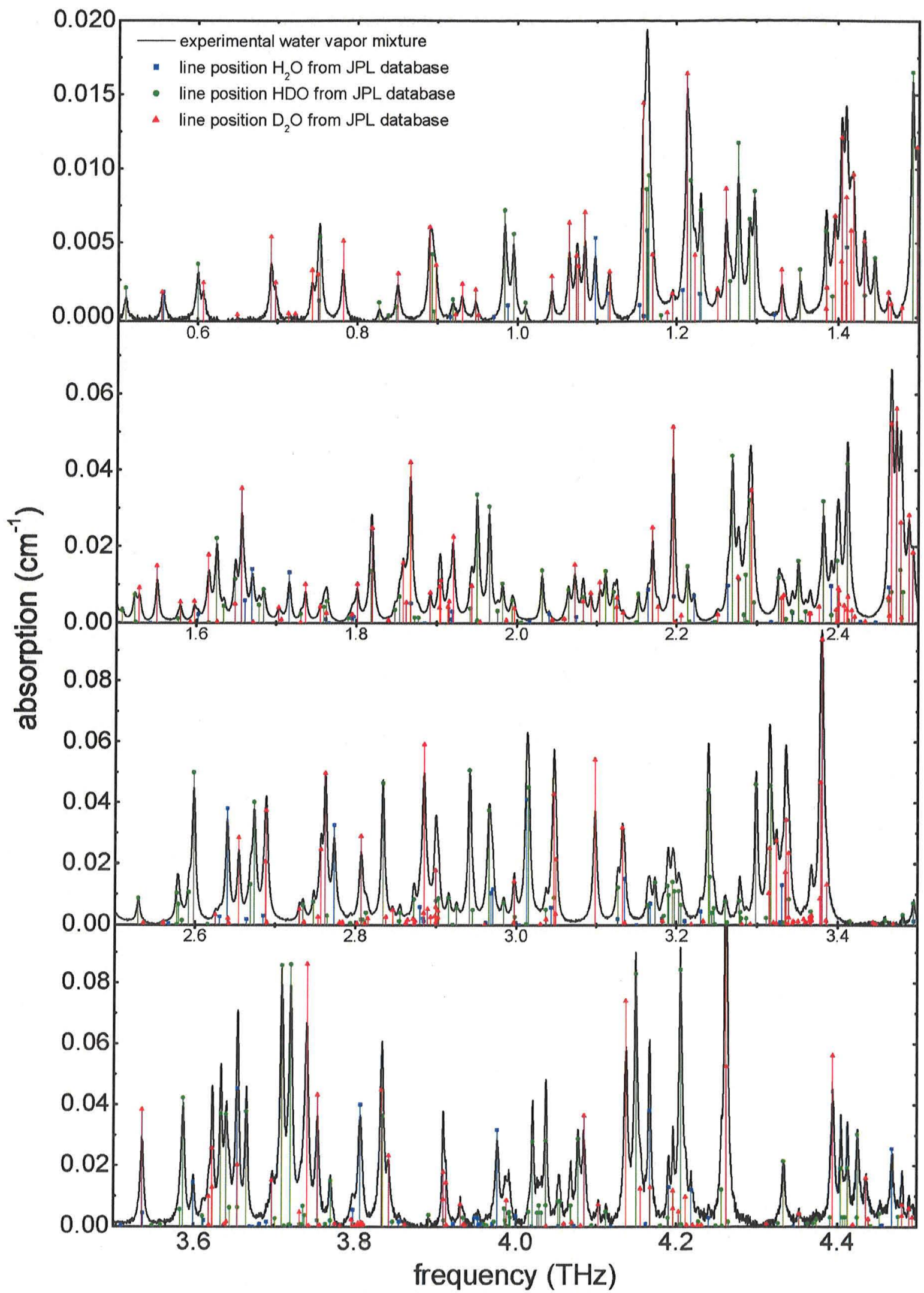

Fig. 6. Detailed absorption spectrum of the experimental water vapor mixture from 0.5 to $4.5 \mathrm{THz}$. Each column covers $1 \mathrm{THz}$. The line positions of $\mathrm{H}_{2} \mathrm{O}$, $\mathrm{HDO}$ and $\mathrm{D}_{2} \mathrm{O}$ are indicated by squares, circles, and triangles respectively and taken from the JPL database [25]. 


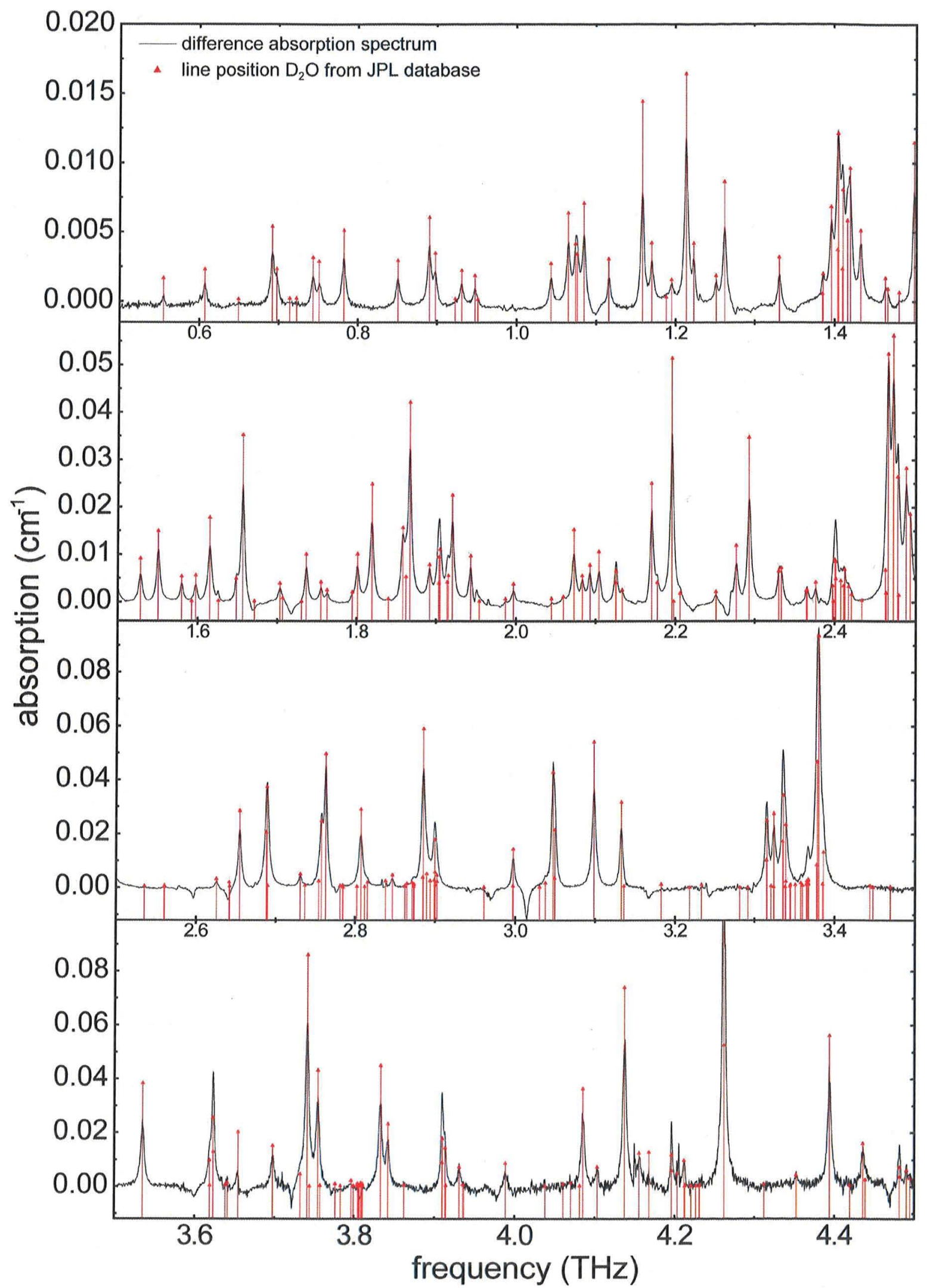

Fig. 7. Detailed absorption spectrum of $\mathrm{D}_{2} \mathrm{O}$ calculated from the experimental data of Fig. 6 by subtracting calculated spectra of $\mathrm{H}_{2} \mathrm{O}$ and $\mathrm{HDO}$. Again the triangles indicate the line positions of $\mathrm{D}_{2} \mathrm{O}$. 

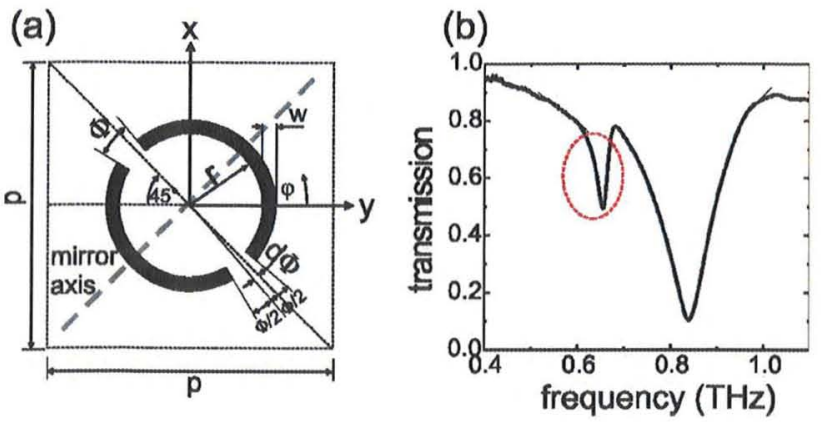

Fig. 8. Lateral unit cell structure of the FSS. The resonators are dimensioned with $w=10 \mu \mathrm{m}, \Phi=22^{\circ}, d \Phi=5^{\circ}, p=197 \mu \mathrm{m}$, and $r=49 \mu \mathrm{m}$. (b) Transmission characteristic of the FSS

\section{FREQUENCY-SELECTIVE SURFACES}

Apart from high-precision gas-phase spectroscopy, another important application of high-speed and high-resolution $\mathrm{THz}$ spectrometer is the rapid and precise readout of characteristic resonances of frequency-selective surface (FSS) sensors in the $\mathrm{THz}$ range. Such devices are based on metallic resonator arrays with tailored transmittance and reflectivity properties [32]. In numerical simulations, it has been proposed to use THz-FSS based on asymmetric split ring resonators as sensor for detecting biomolecular sample films with a thickness of only $10 \mathrm{~nm}$ [33]. Applying substances to an FSS changes the resonant response of the sensor array, which can be monitored in transmittance or reflectivity [34]-[36]. In case of asymmetric split ring resonators, there is an extraordinary strong field enhancement in the gap region of the ring resonator [37]. Compared to classic free-space radiation analysis [38], [39], this field localization leads to a much higher sensitivity of THz-FSS-based sensing reducing the required amount of sample material significantly. For diagnostic applications, however, up to 100 arrayed gene sensors need to be read out in a reasonably short time. Up to now, broad-band sensor readout has been done with rather slow operating conventional $\mathrm{THz}$ time-domain spectrometers based on mechanical delay stages [24]. Thus, we investigated the capability of our high-speed ASOPS spectrometer to precisely determine the resonance of a THz-FSS in a short measurement time.

The THz-FSS consists of asymmetric double-split metallic rings arranged on a quadratic grid with a pitch of $p=197 \mu \mathrm{m}$. A unit cell of the array is shown in Fig. 8(a). The rings are placed on top of a $27-\mu \mathrm{m}$-thick polymer substrate made of benzocyclobutene (BCB) with an assumed permittivity $\varepsilon_{r}=2.6$ and dielectric loss factor $\tan \delta=0.0001$. The FSS is fabricated by spin-on deposition of the BCB layer on a silicon host substrate, including thermal curing at $210^{\circ} \mathrm{C}$ for $2 \mathrm{~h}$. The ring structures are patterned on top of the $\mathrm{BCB}$, using a standard photolithography process, e-beam metal evaporation (Cr/Au with 10-nm/200-nm thickness) and photoresist liftoff. In a last step, the FSS is lifted off the silicon substrate in $\mathrm{KOH}$ solution.

The transmission spectrum of the FSS array is measured with the polarization of the $\mathrm{THz}$ radiation perpendicular to the mirror axis of the FSS in order to excite the highest extinction funda-

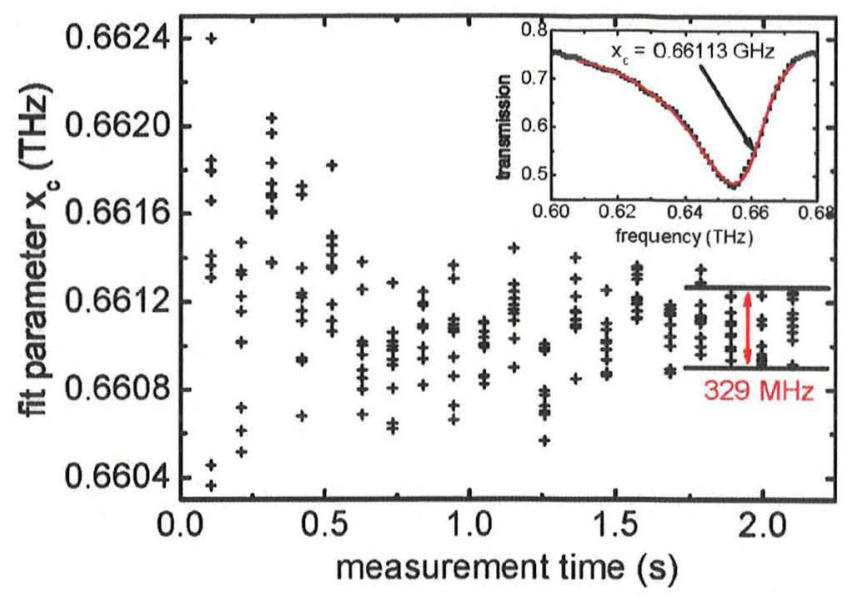

Fig. 9. Fit parameter $x_{c}$ plotted versus measurement time. The inset shows the experimental data (black squares) and the fit function (red solid line).

mental resonance of the FSS [see Fig. 8(b)]. The resonator array is equipped with an element perturbation such that every arc pair provides two slightly different resonant frequencies. Consequentially, the transmission spectrum exhibits the expected devolution with a local transmission maximum in-between a narrow and a broad absorption line generated by modal-interaction as described by Larson and Munk [40]. The local transmission maximum appears when the array self-reactance $X_{A}$ becomes equal to the sum mutual reactance $X_{M}$. In this case, the currents are excited in antiphase. The scattered electromagnetic fields interfere destructively, so the coupling to free space is strongly reduced. Fedotov et al. denote this as a "trapped mode" [41]. For sensing applications, this mode is extremely attractive since it offers strong field localization and temporal remaining energy storage at the resonator surface where thin films of sample material shall be detected.

In order to evaluate the frequency position of the modalinteraction resonance, a function based on two modified sigmoid functions is fitted to the first minimum [see ellipse in Fig. 8(b) and Fig. 9 (inset)]. The function contains five fit parameters

$$
\begin{aligned}
f(x)=y_{0}+A\left(\frac{1}{1+\exp \left(-\frac{x-x_{c}}{w_{1}}\right)}\right) \\
\quad \times\left(1-\frac{1}{1+\exp \left(-\frac{x-x_{c}}{w_{2}}\right)}\right)
\end{aligned}
$$

$y_{0}$ is the offset, $A$ is the Amplitude, $w_{1}$ is the falling edge, $w_{2}$ is the rising edge, and $x_{c}$ describes the position of the resonance [see Fig. 9 (inset)]. Since the fit function is transcendent, the function and its derivatives cannot be analytically solved for $x_{c}$. However, it can be shown that $x_{c}$ must be located between the minimum and the second inflection point. The fit ranges from $0.608-0.673 \mathrm{THz}$ and consists of 55 data points. To test the relation between frequency uncertainty and measurement time, we performed ten individual measurements for different acquisition times ranging from $0.1-2.1 \mathrm{~s}$. In total, 200 spectra were 
fitted. For every fit the parameter $x_{c}$ converges against a fixed value without adjusting the other fit parameters. Fig. 9 shows the fit parameter $x_{c}$ versus measurement time. The scatter of the $x_{c}$ values decreases with measurement time and remains within a $329-\mathrm{MHz}$ band at $1.9 \mathrm{~s}$ or greater. The standard deviation of the acquired data points at $2.1 \mathrm{~s}$ is $108 \mathrm{MHz}$. The statistical fitting error for $x_{c}$ for each individual measurement is about $180 \mathrm{MHz}$. The combination of the standard deviation of $x_{c}$ at $2.1 \mathrm{~s}$ and the statistical fitting error leads to a mean value of $x_{c}=0.66113 \mathrm{THz} \pm 210 \mathrm{MHz}$ within a read-out time of $2.1 \mathrm{~s}$. This result should prove to be highly beneficial for fast sensing of small amounts of biomaterials on a THz-FSS sensor array.

\section{ACKNOWLEDGMENT}

The authors would like to thank C. Kistner, A. Keller, and A. Thoma for their contributions. They would also like to thank S. Winnerl, M. Helm, and A. Leitenstorfer for helpful discussions.

\section{REFERENCES}

[1] M. Tonouchi, "Cutting-edge terahertz technology," Nature Photon, vol. 1, pp. $97-105,2007$.

[2] D. Mittleman, Sensing with Terahertz Radiation. Ser. Springer Series in Optical Sciences. New York: Springer-Verlag, 2003.

[3] H. Harde, R. A. Cheville, and D. Grischkowsky, "Terahertz studies of collision-broadened rotational lines," J. Phys. Chem. A, vol. 101, pp. 3646-3660, 1997.

[4] C. Fattinger and D. Grischkowsky, "Terahertz beams," Appl. Phys. Lett., vol. 54, pp. 490-492, 1989.

[5] X.-C. Zhang, B. B. Hu, J. T. Darrow, and D. H. Auston, "Generation of femtosecond electromagnetic pulses from semiconductor surfaces," Appl. Phys. Lett., vol. 56, pp. 1011-1013, 1990.

[6] R. Huber, A. Brodschelm, F. Tauser, and A. Leitenstorfer, "Generation and field-resolved detection of femtosecond electromagnetic pulses tunable up to 41 THz," Appl. Phys. Lett., vol. 76, pp. 3191-3193, 2000.

[7] A. Sell, R. Scheu, A. Leitenstorfer, and R. Huber, "Field-resolved detection of phase-locked infrared transients from a compact Er:fiber system tunable between 55 and 107 THz," Appl. Phys. Lett., vol. 93, pp. 251107 1-251107-3, 2008

[8] G. Klatt, M. Nagel, T. Dekorsy, and A. Bartels, "Rapid and precise readout of terahertz sensor by high-speed asynchronous optical sampling," Electron. Lett., vol 45, pp. 310-311, 2009.

[9] S. Kim, B. Born, M. Havenith, and M. Gruebele, "Echtzeitnachweis von Änderungen im Protein-Wassernetzwerk während der Proteinfaltung mit Terahertz-Absorptionsspektroskopie," Angewandte Chemie, vol. 120, pp. $6586-6589,2008$.

[10] J. Wosnitza, A. Bianchi, J. Freudenberger, J. Haase, T. Herrmannsdörfer, N. Kozlova, L. Schultz, Y. Skourski, S. Zherlitsyn, and S. Zvyagin, "Dresden pulsed magnctic field facility," J. Magn. Magn. Mater, vol. 310, pp. $2728-2730,2007$.

[11] J. Xu and X.-C. Zhang, "Circular involute stage," Opt. Lett., vol. 29, pp. 2082-2084, 2004.

[12] G.-J. Kim, S.-G. Jeon, J.-I. Kim, and Y.-S. Jin, "Terahertz pulse detection using rotary optical delay line," Jpn. J. Appl. Phys., vol. 46, pp. 7332$7335,2007$.

[13] P. A. Elzinga, R. J. Kneisler, F. E. Lytle, Y. Jiang, G. B. King, and N. M. Laurendeau, "Pump/probe method for fast analysis of visible spectral signatures utilizing asynchronous optical sampling," Appl. Opt., vol. 26 , pp. $4303-4309,1987$.

[14] A. Bartels, R. Cerna, C. Kistner, A. Thoma, F. Hudert, C. Janke, and T. Dekorsy, "Ultrafast time-domain spectroscopy based on high-speed asynchronous optical sampling," Rev. Sci. Instrum., vol. 78, pp. 0351071-035107-8, 2007.

[15] G. Klatt, R. Gebs, C. Janke, T. Dekorsy, and A. Bartels, "Rapid-scanning terahertz precision spectrometer with more than $6 \mathrm{THz}$ spectral coverage," Opt. Exp., vol. 17, pp. 22847-22854, 2009.
[16] C. Janke, M. Forst, M. Nagel, H. Kurz, and A. Bartels, "Asynchronous optical sampling for high-speed characterization of integrated resonant terahertz sensors," Opt. Lett., vol. 30, pp. 1405-1407, 2005.

[17] R. Gebs, G. Klatt, C. Janke, T. Dekorsy, and A. Bartels, "High-speed asynchronous optical sampling with sub-50fs time resolution," Opt. Express, vol. 18, pp. $5974-5983,2010$.

[18] A. Dreyhaupt, S. Winnerl, T. Dekorsy, and M. Helm, "High-intensity terahertz radiation from a microstructured large-area photoconductor," Appl. Phys. Lett., vol. 86, pp. 121114-1-121114-3, 2005.

[19] J. K. Wahlstrand and R. Merlin, "Cherenkov radiation emitted by ultrafast laser pulses and the generation of coherent polaritons," Phys. Rev. B, vol. 68, pp. 054301-1-054301-12, 2003.

[20] D. L. Mills and E. Burstein, "Polaritons: The electromagnetic modes of media," Rep. Prog. Phys., vol. 37, pp. 817-926, 1974.

[21] W. L. Faust and C. H. Henry, "Mixing of visible and near-resonance infrared light in GaP," Phys. Rev Lett, vol. 17, pp. 1265-1268, 1966.

[22] A. Leitenstorfer, S. Hunsche, J. Shah, M. C. Nuss, and W. H. Knox, "Detectors and sources for ultrabroadband electro-optic sampling: Experiment and theory," Appl. Phys. Lett., vol. 74, pp. 1516-1518, 1999.

[23] S. Kasai, T. Katagiri, J. Takayanagi, K. Kawase, and T. Ouchi, "Reduction of phonon resonant terahertz wave absorption in photoconductive switches using epitaxial layer transfer," Appl. Phys. Lett., vol. 94, pp. 113505-1113505-3, 2009.

[24] M. van Exter, C. Fattinger, and D. Grischkowsky, "Terahertz time-domain spectroscopy of water vapor," Opt. Lett., vol. 14, pp. 1128-1130, 1989.

[25] H. M. Pickett, R. L. Poynter, E. A. Cohen, M. L. Delitsky, J. C. Pearson, and H. S. P. Muller, "Submillimeter, millimeter, and microwave spectral line catalog," J. Quant. Spectrosc. Radiat. Transfer, vol. 60, pp. 883-890, 1998.

[26] L. S. Rothman, C. P. Rinsland, A. Goldman, S. T. Massie, D. P. Edwards, J.-M. Flaud, A. Perrin, C. Camy-Peyret, V. Dana, J. Y. Mandin, J. Schroeder, A. Mccann, R. R. Gamache, R. B. Wattson, K. Yoshino, K. V. Chance, K. W. Jucks, L. R. Brown, V. Nemtchinov, and P. Varanasi, "The HITRAN molecular spectroscopic database and HAWKS (HITRAN Atmospheric Workstation): 1996 edition," J. Quant. Spectrosc. Radiat. Transfer, vol. 60, pp. 665-710, 1998.

[27] L. S. Rothman, I. Gordon, A. Barbe, D. Benner, P. Bernath, M. Birk, V. Boudon, L. Brown, A. Campargue, J.-P. Champion, K. Chance, L. Coudert, V. Dana, V. Devi, S. Fally, J.-M. Flaud, R. Gamache, A. Goldman, D. Jacquemart, I. Kleiner, N. Lacome, W. Lafferty, J.-Y. Mandin, S. Massie, S. Mikhailenko, C. Miller, N. Moazzen-Ahmadi, O. Naumenko, A. Nikitin, J. Orphal, V. Perevalov, A. Perrin, A. PredoiCross, C. Rinsland, M. Rotger, M. Simecková, M. Smith, K. Sung, S. Tashkun, J. Tennyson, R. Toth, A. Vandaele, and J. V. Auwera, "The HITRAN 2008 molecular spectroscopic database," J. Quant. Spectrosc. Radiat. Transfer, vol. 110, pp. 533-572, 2009.

[28] P. D. Bièvre, M. Gallet, N. E. Holden, and I. L. Barnes, "Isotopic abundances and atomic weights of the elements," J. Phys. Chem. Ref. Data, vol. 13, pp. 809-891, 1984.

[29] B. Yu, Y. Yang, F. Zeng, X. Xin, and R. Alfano, "Terahertz absorption spectrum of $\mathrm{D}_{2} \mathrm{O}$ vapor," Opt. Commun., vol. 258, pp. 256-263, 2006.

[30] R. Paso and V.-M. Horneman, "High-resolution rotational absorption spectra of $\mathrm{H}_{2}^{16} \mathrm{O}, \mathrm{HD}^{16} \mathrm{O}$, and $\mathrm{D}_{2}^{16} \mathrm{O}$ between 110 and $500 \mathrm{~cm} 1$," J. Opt. Soc. Amer: B. Opt. Phys., vol. 12, pp. 1813-1838, 1995.

[31] J. Wolk and R. Strey, "Homogeneous nucleation of $\mathrm{H}_{2} \mathrm{O}$ and $\mathrm{D}_{2} \mathrm{O}$ in comparison: The isotope effect," J. Phys. Chem. B, vol. 105, pp. 11683$11701,2001$.

[32] B. A. Munk, Frequency Selective Surfaces: Theory and Design. New York: Wiley-Interscience, 2000.

[33] C. Debus and P. H. Bolivar, "Frequency selective surfaces for high sensitivity terahertz sensing," Appl. Phys. Lett., vol. 91, pp. 184102-1-184102-3, 2007.

[34] R. Singh, E. Smirnova, A. J. Taylor, J. F. O'Hara, and W. Zhang, "Optically thin terahertz metamaterials," Opt. Exp., vol. 16, pp. 6537-6543, 2008.

[35] H. Yoshida, Y. Ogawa, Y. Kawai, S. Hayashi, A. Hayashi, C. Otani, E. Kato, F. Miyamaru, and K. Kawase, "Terahertz sensing method for protein detection using a thin metallic mesh," Appl. Phys. Lett., vol. 91, pp. 253901-1-253901-3, 2007.

[36] J. F. O'Hara, R. Singh, I. Brener, E. Smirnova, J. Han, A. J. Taylor, and W. Zhang, "Thin-film sensing with planar terahertz metamaterials: Sensitivity and limitations," Opt. Exp., vol. 16, pp. 1786-1795, 2008.

[37] I. A. I. Al-Naib, C. Jansen, and M. Koch, "Thin-film sensing with planar asymmetric metamaterial resonators," Appl. Phys. Lett., vol. 93, pp. 083507-1-083507-3, 2008. 
[38] M. Brucherseifer, M. Nagel, P. H. Bolivar, H. Kurz, A. Bosserhoff, and R. Büttner, "Label-free probing of the binding state of DNA by timedomain terahertz sensing," Appl. Phys. Lett., vol. 77, pp. 4049-405I, 2000.

[39] T. Globus, D. Woolard, T. W. Crowe, T. Khromova, B. Gelmont, and J. Hesler, "Terahertz Fourier transform characterization of biological materials in a liquid phase," J. Phys. D: Appl. Phys., vol. 39, pp. 3405-3413, 2006.

[40] C. Larson and B. Munk, "The broad-band scattering response of periodic arrays," IEEE Trans. Antennas Propag., vol. AP-31, no. 2, pp. 261-267, Mar. 1983.

[41] V. A. Fedotov, M. Rose, S. L. Prosvirnin, N. Papasimakis, and N. I. Zheludev, "Sharp trapped-mode resonances in planar metamaterials with a broken structural symmetry," Phys. Rev. Lett., vol. 99, pp. 147401-1$147401-4,2007$

Gregor Klatt received the Diploma degree in physics in 2007 from the University of Konstanz, Konstanz, Germany, where he is currently working toward the Ph.D. degree at the Department of Physics and the Center for Applied Photonics. His current research interests include terahertz science and technology, the development of new high-power terahertz emitters, and high-speed and highresolution terahertz spectroscopy.

Raphael Gebs received the Diploma degree in physics in 2007 from the University of Konstanz, Germany, where he is currently working toward the Ph.D. degree at the Department of Physics and the Center for Applied Photonics.

His current research interests include time-domain spectroscopy based on ultrafast asynchronous optical sampling, especially the improvement of its time resolution, and possible new applications of it.

Hanjo Schäfer received the Diploma degree in 2007 from the University of Konstanz, Germany, where he is currently working toward the Ph.D. degree at the Department of Physics and the Center for Applied Photonics.

$\mathrm{He}$ is currently engaged in research on investigating strongly correlated electron systems with the help of ultrafast optical spectroscopy in the THz, IR, and visible range and the ultrafast electron diffraction technique.

Michael Nagel received the Ph.D. degree from the Rheinisch-Westfalische Technische Hochschule (RWTH) Aachen University, Aachen, Germany, in 2003.

During his thesis. he developed the first label-free DNA sensor chip utilizing $\mathrm{THz}$ range frequencies. Since 2004, he has been the Chief Engineer at the Institute of Semiconductor Electronics, RWTH Aachen University, where he directs applied and fundamental research projects focusing on terahertz technology $\mathrm{He}$ is the author or coauthor of more than 80 papers published in international journals and conference proceedings. He is the holder of six patents. His current research interests include terahertz technology developments in the range of integrated device realization and characterization, near-field imaging, biosensing. and silicon nanophotonics.

Dr. Nagel was the recipient of the Roberts Prize 2004 of the Institute of Physics and Engineering in Medicine and the Wilhelm Borchers Medallion of the RWTH Aachen University.
Christof Janke received the Diploma degree and Ph.D. degree in physics from the Rheinisch-Westfalische Technische Hochschule (RWTH) Aachen University, Aachen, Germany, in 1999 and 2004, respectively.

During his Ph.D. studies, he was engaged in research on THz science and technology in combination with high-repetition-rate femtosecond Ti:sapphire lasers. He is currently with Gigaoptics GmbH, Konstanz, Germany, and also a Researcher at the Department of Physics and the Center for Applied Photonics, University of Konstanz, Konstanz, Germany.

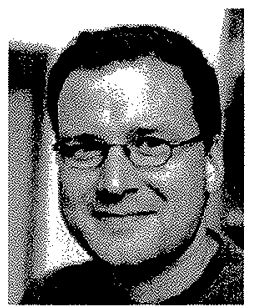

Albrecht Bartels received the Diploma degree and Ph.D. degree in physics from the RheinischWestfälische Technische Hochschule (RWTH) Aachen University, Aachen, Germany, in 1997 and 2000 , respectively.

During his Ph.D. studies, he developed the first Ti:sapphire femtosecond laser with repetition rates above $1 \mathrm{GHz}$. During his postdoctoral positions at RWTH Aachen University from 2000 to 2001 and at the Time and Frequency Division of the National Institute of Standards and Technology, Boulder, $\mathrm{CO}$, from 2001 to 2004, he explored possibilities to use gigahertz repetition rate femtosecond lasers for the generation of ultrastable optical frequency combs. $\mathrm{He}$ is currently the Chief Executive Officer at Gigaoptics GmbH, Konstanz, Germany, and a Junior Research Group Leader at the Department of Physics and the Center for Applied Photonics, University of Konstanz, Konstanz, Germany. His research interests include ultrafast lasers, optical frequency combs, and novel methods for time-domain terahertz and pump-probe spectroscopy

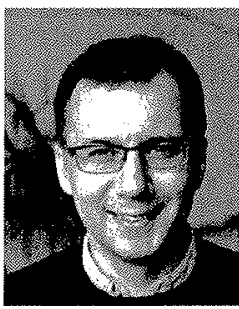

Thomas Dekorsy received the Diploma degree in physics and the Ph.D. degree from the RheinischWestfälische Technische Hochschule Aachen University, Aachen, Germany, in 1993 and 1996 respectively.

In 2000, he was the Head at the Research Center Dresden-Rossendorf, Germany, where he newly established a Semiconductor Spectroscopy Division and contributed to the first lasing of the THz-freeelectron laser (FEL) facility FELBE. Since 2005 , he has been a Full Professor in the Department of Physics and the Deputy Director of the Center for Applied Photonics at the University of Konstanz, Konstanz, Germany. He is the author or coauthor of more than 100 papers published in the field of uttrafast spectroscopy and $\mathrm{THz}$ technology. 